\title{
THE EFFECTS OF STIMULUS VARIABILITY AND SIGNAL VALUE UPON HABITUATION OF THE ORIENTING RESPONSE'
}

\author{
YOSHINORI MITSUHASHI ${ }^{2}$, TETSURO MINO, AND YO MIYATA \\ Kzonsei Gakuin University
}

\begin{abstract}
The effects of stimulus variability and task upon the habituation of the skin resistance response component of the orienting response (OR), and upon ORreturn elicited by novel stimuli were examined. Six groups were designed according to three variability conditions and two task conditions. Larger magnitude of OR and slower habituation were found for higher variability and task conditions. Moreover, lower variability produced a larger OR-return. These results were discussed in terms of signal value and uncertainty as proposed by Sokolov's theory $(1963,1969)$.
\end{abstract}

Since the proposal of Sokolov's neuronal model theory (1963), the orienting response (OR) and its habituation have continued to hold the interest of researchers. Miyata, and co-workers (Mino \& Miyata, 1975; Miyata, Mino, \& Mizuno, 1977) performed a series of experiments concerning the effects of the range of stimulus variability in the intensity-dimension upon the skin resistance response (SRR) component of OR. They failed to show any significant effects on the habituation period, contrary to Sokolov's theory, but a larger OR-return was shown in a lower variability condition by presenting novel stimuli.

Two questions were raised, however, in their experiments. Firstly, could the same results be expected for the other stimulusdimension? Unfortunately, there have been only a few studies on this theme, with

1 A part of this study was supported by a Grant in Aid for Scientific Researches, Ministry of Education.

${ }^{2}$ The authors are grateful to Prof. M.E. Reames for his many helpful suggestions in the revision of this article. Request for reprints should be sent to Yoshinori Mitsuhashi, Department of Psychology, Kwansei Gakuin University, 1 Uegahara, Nishinomiya, Hyogo, 662 Japan. contradictory findings (Ely, 1972; Kimmel, 1973). The second question concerns the effect of the subject's set or attentional state to stimulus. Mitsuhashi, Mino, Mizuno, and Miyata (1974) reported in their review that making a subject perform a task to stimulus retards habituation of OR. This procedure, "signalization", may have a potential influence on the effect of stimulus variability as well.

Concerning the interactive effects of task and stimulus variability upon the habituation of $\mathrm{OR}$, there have also been a few studies. Ray, Piroch, and Kimmel (1977), in their second experiment, revealed some interactions of the task by variability in the duration-dimension of tone stimuli, i.e., slower habituation of the SRR component of OR was shown for a higher variability condition in a nontask situation, and for a lower condition in the reaction time-task situation. We have, however, some comments on their experiment. Firstly, only two conditions were selected from the variability dimension. Secondly, the range of stimulus variability was too narrow, i.e., 4 to $8 \mathrm{~s}$. Thirdly, they didn't investigate the effects 
on the OR-return elicited by novel stimuli. Finally, the response to the tone offset was not analyzed in spite of the fact that reaction time to the tone offset was measured.

In order to solve the above-mentioned problems, the present study re-examined the effects of task and stimulus variability in the tone duration-dimension upon the habituation and OR-return.

\section{MEthoD}

\section{Subjects}

Seventy-two undergraduates (39 males \& 33 females), with a mean age 19.3 years, ranging in age from 18 to 24 years, served as subjects. They were randomly assigned to six groups of 12 subjects.

\section{Apparatus}

Stimulus. The auditory stimulus was a $1000 \mathrm{~Hz}$ pure tone produced by an audio generator (Lead Model LAG-66) and presented to subject via headphone. Tone intensity was $75 \mathrm{~dB}$ in the habituation trials and $90 \mathrm{~dB}$ in the test trials, both as rated by a sound level meter (Rion NA07A) in C-scale at the headphone.

Response. SRR was taken from the palm of the subject's left hand by AgAgCl electrodes (Nihon Koden Type-25) mounted in bakelite cups filled with $\mathrm{NaCl}$ electrolyte. Through a Wheatstone bridge circuit the SRR was amplified in a DCcoupler and recorded by a pen-galvanometer (Sanei Biophysiograph 180 system). Electromyographs of subject's right forearm (.03 s time constant) and respiration were recorded concomitantly with the SRR, but they were not quantitatively analyzed in the present study.

\section{Design}

The six groups were designed according to two experimental factors, i.e., variability and task. The variability factor consisted of three conditions (1D, 2D, \& 4D), defined by the combination with stimulus-duration in the habituation trials. Four durations, 5, 7.5, 10, and $12.5 \mathrm{~s}$, were used. One duration was selected for the ID condition, two for the 2D condition, and all four durations for the 4D condition. They were randomly scattered over the trials. Possible combinations of duration were counterbalanced among the subjects. The task factor was composed of two conditions, reaction timetask (RT) condition and non-task (NT) condition. For the RT condition subject was asked to push a button with his right finger at the offset of the stimulus as quickly as possible, and for the NT condition subject was asked to relax and to avoid movement.

\section{Procedure}

Subject seated in a reclining chair in a sound-attenuated room, was informed that some tones would be heard from time to time and that the experiment was to record his physiological response to sound stimuli. Half of the subjects (RT condition), moreover, were given instructions concerning a reaction time-task. After a $3 \mathrm{~min}$ rest period, 16 habituation trials and nine test trials were started without a prior warning. In the habituation trials $75 \mathrm{~dB}$ tones with durations according to each variability condition were presented and in the test trials the tone intensity was increased to $90 \mathrm{~dB}$. Throughout all the trials the button press task was requested for RT condition subjects. Interstimulus interval was from 40 to $60 \mathrm{~s}$, with a mean interval of $50 \mathrm{~s}$.

\section{Data Analysis}

Two SRR magnitude measures were scored in each trial, i.e., the response to the stimulus onset, initiated within .5 to $5 \mathrm{~s}$ following the tone onset (ON-response), and the response to the stimulus offset, within .5 to $5 \mathrm{~s}$ following the tone offset (OFF-response). Both were changes in skin resistance of more than $0.5 \mathrm{k} \Omega$ and transformed by the square root 
of the conductance change $(\sqrt{\Delta \mathrm{C}})$.

\section{Results}

\section{ON-response}

Habituation trials. Figure 1 presents the averaged SRR magnitude by blocks of four trials for the habituation trials. An analysis of variance (Type III, Lindquist, 1956) indicated that the effects of task $(F(1,66)=28.35, p<.001)$, of variability $(F(2,66)=5.02, \quad p<.05)$, and of blocks $(F(3,198)=67.80, p<.001)$ were all significant. As for their interactions there were no significant effects. Further analysis of variance (Type I, Lindquist, 1956) for the first trial revealed a significant task effect $(F(1,66)=10.08, p<.005)$, but no effects of either variability or interaction were proved. Median habituation speed, i.e., the number of trials untill three successive non-responses, of the 1D, 2D, and $4 \mathrm{D}$ groups is 4,8 , and 10 , respectively, in NT condition, and 13,16, and 16 in RT condition. The Krustal-Wallis $H$ test revealed both significant task and signifcant variability effects. A separate analysis for variability effect, however, indicated significant differences in the habituation speed only in the RT condition.

Thus, a larger magnitude and slower habituation speed of the $O R$ during habituation trials were found for the RT

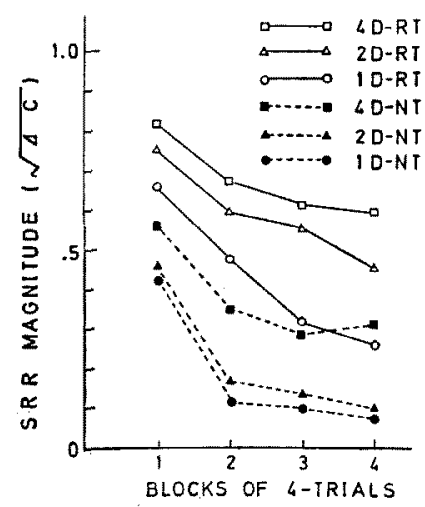

FIG. 1. Mean SRR magnitude of the ON-response for six groups in the habituation trials. condition than the NT condition. Moreover, higher stimulus variability retarded habituation of the OR, though the effect was ambiguous in the NT condition.

Test trial. In the first test trial the increase of tone intensity to $90 \mathrm{~dB}$ augmented the SRR magnitude for all six groups. Because of the significant differences during habituation trials, all SRRs in the test trials were divided by those in the last habituation trial, and then transformed to the SRR ratio. Figure 2 presents the averaged SRR ratio by blocks of 2 trials except for the first trial (TS-1). An analysis of variance (Type III) indicated that the effects of variability $(F(2,66)=8.23, p<.001)$, of blocks $(F(4,264)=36.09, p<.001)$ and of variability by block-interaction $(F(4,264)=$ $2.42, p<.05)$ reached significant levels. Further analysis revealed significant differences among groups in SRR ratio through TS-1 up to the second block. Thus, the novel stimulus brought a more remarkable enhancement of the OR for the lower variability condition regardless of the task condition.

\section{OFF-response}

Habituation trials. Averaged SRR magnitude to the tone offset showed a prominent task effect over all trials. Larger magnitude for the RT condition was

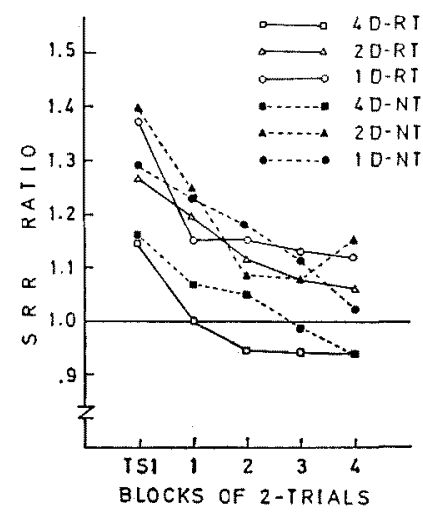

Fig. 2. Mean SRR ratio of the ON-response for six groups in the test trials. 
found through the habituation trials than for the NT condition. An analysis of variance (Type III) for blocks of four trials indicated that there were significant effects of task $(F(1,66)=84.96, p<.001)$ and blocks $(F(3,198)=8.30, p<.001)$, but the effects of variability did not reach a critical level $(F<1)$.

Test trials. There was no tendency toward OR-return for the first test stimulus for all six groups. Although OFF-response in the test trials were transformed to SRR ratio as for ON-responses, there were no effects either of variability or of task, except a significant block effect $(F(4,264)=3.96, p<.025)$. Thus, in the OFF-response, OR was increased for the RT condition, but stimulus variability had no effect upon either habituation or ORreturn.

\section{Discussion}

The results of the present study showed that a differential habituation occurred in both variability and task conditions, except for the effect of variability upon $\mathrm{OFF}$-response.

Making a subject perform a reaction time-task augmented both ON- and OFFORs and retarded habituation. The results of OFF-OR are a matter of course, because the muscular activity itself should maintain larger and more persistent ORs. In the case of ON-OR, however, the effect of task can not be attributed to motor activity. As the fundamental relationship between SRRs and motor activity is still unknown (van Olst, 1971), it is plausible to think that the effect of task on OR-habituation is to add a functional significance, i.e., "signal value", to the stimulus as suggested by Bernstein (1969).

Stimulus variability is a critical factor for $O R$ and its habituation. The higher the variability was, the slower the habituation of the ON-OR was found to be, though the effect was ambiguous in the NT condition. These results were in- consistent with Ray et al. (1977). They suggested from the standpoint of two feedback reflexes in conditioning (Kimmel, 1973) that habituation is a curvilinear function of task difficulty, i.e., a more difficult task resulted in less retardation of habituation. Their explanation is implausible for the present data, for a more difficult task than theirs was used and the results revealed a linear function. Retardation of habituation may be rather a direct function of stimulus variability, and the task will have a facilitating effect upon this function.

Present results confirm Sokolov's theory (1963), but it is only acceptable for ONOR. In the case of OFF-OR there were no effects of variability, and this contradicts the match-mismatch model. For mismatch may occur at the offset of each stimulation. Sokolov (1969) modified his model from the standpoint of information theory and suggested that $O R$ is a function of uncertainty of the stimulus. If it is the case, the present results may be acceptable, for uncertainty may still remain at the onset of each stimulation, and information of the critical parameters is presented only at the offset of a stimulus with variable duration. Thus, uncertainty might be a critical factor for ORhabituation. The results of test trials indicated that OR-return was an inverted linear function of variability. This is consistent with our previous findings (Mino \& Miyata, 1975) and with Sokolov's theory (1963, 1969).

\section{REFERENCES}

Bernstein, A.S. 1969 To what the orienting response respond? Psychophysiology, 6, 338-350.

ELY, D. J. 1972 Temporal duration as a dimension in generalization of the orienting response. Perceptual and Motor Skills, 34, 271-276.

KrmmeL, H. D. 1973 Habituation, habituability, and conditioning. In H. V. Peeke \& M. J. Herz (Eds.), Habituation. Vol. 1: Behavioral studies. New York: Academic Press, Pp. 219-238. LINDQuist, E. 1956 Design and analysis of experi- 
ments in psychology and education. Boston: Houghton Mifflin.

Mino, T., \& Miyata, Y. 1975 Effects of the range of stimulus-variation upon habituation of the orienting response. Psychophysiology, 12, 136-140.

Mryata, Y., Mino, T., \& Mizuno, K. 1977 Effects of the range of stimulus-variation upon habituation of the orienting response: Further demonstrations under extreme stimulus-conditions. Kwansei Gakuin Universily Annual Studies, 27, 115-120.

Mitsuhash, Y., Mino, T., Mizuno, K., \& MiyaTA, Y, 1974 Habituation of the autonomic components of orienting reflex: A review.
Japanese Psychological Review, 17, 179-202. (In Japanese).

van OLst, E. H. 1971 The orienting reflex. The Hague: Mouton.

Ray, R. L., Piroch, J. E., \& Kimmel, H. D. 1977 The effect of task and stimulus variability on habituation of electrodermal and vasomotor reactions. Physiological Psychology, 5, 189-196.

Soxolov, E. N. 1963 Perception and the conditioned reflex. New York: Pergamon Press.

Sokolov, E. N. 1969 The modeling properties of the nervous system. In M. Coles \& I. Malzman (Eds.), $A$ handbook of contemporary Soviet psychology. New York: Basic Books.

(Received June 11, 1979) 